\title{
An updated review of the evidence for adjustable compression wrap devices in the lower limb.
}

\author{
Accepted $7^{\text {th }}$ September 2017
}

Dr Anne Williams

Lecturer and Lymphology Nurse Consultant

Queen Margaret University

Contact address:

Queen Margaret University

Queen Margaret Drive

Edinburgh

EH21 6UU

Email: awilliams@qmu.ac.uk

Key words: adjustable compression wrap devices; compression therapy; lymphoedema; chronic oedema; lipoedema; venous ulceration 


\begin{abstract}
Compression therapy is a key component in the effective management of people with lower limb problems associated with venous, lymphatic and fat disorders such as lipoedema. Individuals with lymphoedema, venous ulceration and lipoedema often require long term compression therapy to prevent and manage problems such as chronic ulceration and skin changes, persistent swelling and shape distortion. Challenges remain in achieving acceptable, safe, effective and cost-efficient compression therapy choices. ${ }^{5}$ Adjustable compression wrap devices using hook and loop fasteners, commonly called VELCRO brand fasteners, present new opportunities for improving treatment outcomes, supporting patient independence and self-management in the use of compression therapy. This paper reports the findings of an evidence review of adjustable compression wrap devices in people with lymphoedema, chronic oedema, venous ulceration and lipoedema.
\end{abstract}

\title{
Introduction
}

There is a relatively wide choice of compression therapy products and devices currently available in the UK. ${ }^{1}$ These include a variety of elastic and inelastic compression bandaging systems, circular and flat-knit hosiery, and adjustable compression (VELCRO) wrap devices. A skilled person-centred assessment underpins treatment choices in compression therapy, taking into account the pathophysiology of a condition, symptoms experienced by the person, their social and personal context, and the treatment aims. Compression therapy has various effects such as reducing capillary filtration, minimising accumulation of tissue fluid and inflammatory processes, enhancing venous return, and improving lymphatic transport capacity. ${ }^{2}$ Compression therapy promotes venous ulcer healing. ${ }^{3}$ It also reduces limb volume and enhances physical functioning in those with lower limb lymphoedema. ${ }^{4}$ Individuals using compression garments are less likely to report pain, numbness and poor range of movement than those not using compression. ${ }^{5,6}$ Self-application of compression is reported as providing greater independence, self-efficacy, and a greater sense of control for people with lymphoedema. ${ }^{7}$ 
Intensive treatment using compression therapy can be demanding for patients and professionals, particularly if regular bandage application is necessary due to severe ulceration, leakage of exudate, or problematic skin conditions. Miller et al. ${ }^{8}$ described more than $50 \%$ of patients treated with multi-layer bandaging as non-concordant, for various reasons such as poor tolerance of treatments or limited motivation. Furthermore, the transition from one type of compression therapy such as regular bandaging, to wearing compression stockings, can be challenging ${ }^{9}$ due to difficulties in applying compression stockings, deterioration in skin condition, or rebound oedema. This suggests that choice is required in compression therapy, to better address clinical problems, and achieve costeffective treatment outcomes. Advances in textile technology have led to the development of a range of adjustable compression wrap devices that are suitable for people with people with lymphoedema, chronic oedema, venous ulceration and lipoedema in the intensive treatment and maintenance phases.

\section{Adjustable compression wrap devices}

The devices consist of low-elastic material sections that wrap across the limb and are secured with hook and loop fasteners. They are designed for self-application over the short or long term, enabling the patient or carer to apply or adjust the device as required. ${ }^{10-12}$ Several product types are described in the literature, including the CircAid, the Juxta-Fit and JuxtaCURES systems from medi UK, the Jobst FarrowWrap, the Haddenham easywrap, and the ReadyWrap adjustable compression garment from Lohmann and Rauscher. The devices are available in different sizes, and various foot piece, leg piece or thigh piece sections. Some are available in different fabrics, to suit contrasting patient groups such as those with severe lymphoedema who require stronger materials, or those with more palliative needs who require lighter compression. Most devices are available on prescription and some can be custom-made, or available to cut and reshape according to individual patient need. There is some indication that adjustable compression wraps may achieve similar subgarment pressure profiles to short stretch bandaging systems. ${ }^{13,14} \mathrm{It}$ is suggested that they create a semi-rigid compression system that provides resistance to tissue movement, enabling pressure variations within the tissues, and thereby promoting healthy lymphatic and venous function. ${ }^{15}$ 


\section{Aims of the review.}

The review aimed to address the following questions:

- What is the clinical evidence for the use of adjustable compression wrap devices in people with lower limb conditions such as venous ulceration, chronic oedema, lymphoedema and lipoedema?

- What are the economic implications of using these devices?

The review also aimed to identify recommendations and future directions for practice and research.

\section{Methodology}

Databases used: Medline; CINAHL; PsycINFO; Cochrane Library; Joanna Briggs Institute. Google Scholar was also searched, conference posters and oral presentations were included where available online, and citations on individual papers were also checked.

Search terms: VELCRO Compression Wraps; compression-wraps; CircAid; Juxta-Fit;

JuxtaCURES; FarrowWrap; Haddenham easywrap; ReadyWrap were combined with: venous ulceration; chronic oedema; lymphoedema; lymphedema; lipoedema; lipedema. Inclusion criteria: January 1996-July 2017; research studies; review papers; Consensus and Best Practice Documents and Guidelines; descriptive papers; conference papers and posters available online.

Exclusion criteria: papers not in English language.

Search results: a total of 72 articles were identified with 33 meeting the inclusion criteria for the review. There were no review papers, consensus or best practice guidelines on the topic, and no papers evaluating the use of adjustable compression wraps in people with lipoedema.

\section{Use and clinical effects of adjustable compression wrap devices in people with chronic oedema or lymphoedema}

The first description of an adjustable compression wrap product in the UK literature was the CircAid, developed in the US, and presented in three case studies by a lymphoedema practitioner. ${ }^{16}$ The Juxta-Fit product was presented in lymphoedema case studies, illustrating its' use as a stand-alone garment or for use in conjunction with compression 
garments. ${ }^{10,17}$ The device is described as providing sustained pressure using the 'Juxta-lock' system of interlocking bands that can be loosened or tightened. ${ }^{10}$

The ReadyWrap adjustable compression wrap system was reported in conference posters from the USA as being well tolerated and reducing limb volume in three patients with bilateral lower limb oedema ${ }^{18}$, with further evidence that the device has potential to improve function, gait and balance ${ }^{19}$. TO HERE A series of descriptive papers discussed the FarrowWrap system for lymphoedema, originally brought to the UK market by Haddenham Healthcare. ${ }^{11,16-19}$ FarrowWrap is an adjustable hook and loop fastener system with multiple overlapping neoprene bands, interconnected by a spine. A case study described its' use in a young women with low mood who was not keen to have intensive bandaging treatment. ${ }^{16}$ Hobday and Wigg ${ }^{19}$ describe the product as encouraging practitioners to take a creative, problem-solving approach to care, working in partnership with the patient. Both devices are reported as versatile and easily adjusted to a change in limb size to enable selfmanagement of oedema reduction ${ }^{10,18}$, while also allowing for skin care to be carried out on a regular basis. ${ }^{19}$ FarrowWrap has also been suggested as a useful garment for night use and compared favourably to short stretch bandaging ${ }^{20}$, although research evidence is lacking and the above papers are all descriptive in nature with only two research studies identified.

In the first research paper, Damstra and Partsch ${ }^{21}$ undertook a prospective, randomised controlled trial of 30 hospitalised patients with unilateral primary or secondary lymphoedema of the leg (Stage 2-3). In one group, 15 patients with a mean age of 54.5 years had the Adjustable Compression Wrap (ACW) applied at Time 0 by the clinician, removed at two hours (Time 2 ) and replaced by the patient. The ACW was then worn for a further 22 hours (until Time 24) during which time the participants were instructed to adjust it as required. A second group of 15 patients with a mean age of 59.9 years wore inelastic multi-component compression bandages on the leg, removed and replaced by staff after two hours, and then worn for a further 22 hours. The researchers measured reduction in leg volume (using the water displacement method), calculating the reduction in excess limb volume, and also recorded interface pressures using a PicoPress monitor. ${ }^{21}$ 
The mean volume reduction at two hours was not significantly different between the two groups. However, the difference was significant after 24 hours; the group wearing ACW had a mean $339 \mathrm{mls}(-10.3 \%)$ reduction in excess limb volume, compared to a mean $190 \mathrm{mls}$ ($5.9 \%)$ reduction in the bandaged group $(p<0.05)$. Mean interface pressures in the ACW group were $53 \mathrm{mmHg}$ at initial application and $35 \mathrm{mmHg}$ at two hours (26.1\% reduction). ${ }^{21}$ In comparison, the mean interface pressure in the bandaging group was $49 \mathrm{mmHg}$ at initial application and $27 \mathrm{mmHg}$ at two hours (50\% reduction). ${ }^{21}$ Patient reapplication of the ACW at two hours achieved a mean interface pressure of $52 \mathrm{mmHg}$, very similar to the initial nurse application pressures at Time 0 . At 24 hours, median interface pressure decreased significantly to $33 \mathrm{mmHg}$ in the ACW group, compared to $25 \mathrm{mmHg}$ in the bandaging group $(p<.001)$. The researchers concluded that the ACW system achieved a greater reduction in limb volume than the bandaging system. Furthermore, the ability to adjust the garment, and evidence that patient reapplication achieved similar interface pressures as the health professional, led the authors to suggest that the adjustable compression wrap product was suitable for self-management. However, outcomes were limited to a 24 hour period, and details of clinical effectiveness or patient comfort over a longer period were not reported. ${ }^{21}$

In the second research paper, Mosti et al..$^{22}$ compared the efficacy and comfort of inelastic bandaging (IB) and an Adjustable Velcro Compression Device (AVCD) in 36 patients (40 legs) with untreated venous oedema due to superficial or deep venous insufficiency. Patients had a mean age of 71.4 years. One group was randomised to a week of treatment with a short stretch bandaging system applied in a spiral method to achieve a supine sub-bandage pressure of $60 \mathrm{mmHg}$ (as measured by the PicoPress). ${ }^{22}$ The second group was treated with the Juxta-Fit garment applied to achieve a pressure of $40 \mathrm{mmHg}$ when supine, with the instruction to adjust the device if they became aware of a decrease in the compression. Leg volume was calculated using the truncated cone formula (at $4 \mathrm{~cm}$ interval circumferences along the limb) at Time 0, Day 1 (Time 1) and Day 7 (Time 7). Reduction in volume was calculated in millilitres and expressed as a percentage of the initial limb volume. Interface pressures were measured at the B1 point using the PicoPress monitor. Measures of comfort were completed at Time 1 and 7 using visual analogue scales to subjectively assess symptoms such as pain, heaviness, swelling sensation, discomfort, itching and restless leg using scores from 0-10. Subjective measures such as wearing a shoe, ease of application 
and readjustment at T0, T1 and T7 were similarly scored. The sum of scores was calculated to provide a comfort index and an index of other parameters. ${ }^{22}$

The researchers reported that mean interface pressures were higher in the bandaging group than the AVCD group at supine and standing at application; however, at Time 1 (24 hours) this was reversed. Mean volume reduction at Time 1 and 7 was significantly higher in the AVCD group than the bandaging group $(p>.001)$. However, it is important to note that the method used for calculating change in limb volume as a percentage of the initial limb size may be less accurate than monitoring change in excess volume when compared to an unaffected limb. ${ }^{23}$ Comfort scores decreased in both the bandaging and AVCD groups. ${ }^{22}$ Patients found reapplication of the AVCD relatively easy, and scored this more favourably for cosmetic appearance and ease of putting on shoes than the bandaging system. The researchers concluded that the AVCD was ideal for self-management as it is easily applied and adjusted correctly by the patient following a simple teaching session. ${ }^{22}$ Furthermore, they identified that interface pressures were able to be maintained as the device was readjusted, unlike bandaging systems where pressures drop once oedema reduces. Limitations of the product were also reported, for example, difficulties with application when someone is overweight and inflexible, or there is severe shape distortion on the $\operatorname{limb} .22$

\section{Adjustable compression wrap devices in venous ulcer management}

Lawrence ${ }^{24}$ described the JuxtaCURES as an innovative method for leg ulcer management, combining a liner, anklet and legging garment that can be adjusted, particularly useful for self-management and when a leg is too large to be accommodated in a leg ulcer kit. DePalma et al..$^{25}$ undertook a comparative study of CircAid (Theraboot or TB) and the Unna Boot (UB) in 28 patients with small leg ulcers eligible for conservative treatment, across six community centres in the USA. The mean time to healing in the UB group (11 patients) was 9.69 weeks and the TB group (17 patients) was 7.98 weeks, with a non-statistically significant trend $(p=0.41)$ to faster healing times evident in the TB group. ${ }^{25}$ In another trial, 12 patients (mean 61 years) with post-thrombotic bilateral lower limb ulceration were randomised to treatment with four layer elastic bandaging to one $\operatorname{limb}$, and the CircAid adjustable compression wrap to the contralateral limb. ${ }^{26}$ A significantly faster healing rate 
was described under the adjustable compression wrap, than the bandaging system $(p=$ .0173), although the patients were not monitored beyond 12 weeks. ${ }^{26}$

Various authors have used case studies to showcase the products and explore outcomes. Bock ${ }^{27}$ described the use of the ReadyWrap adjustable compression garment in three patients with chronic venous ulceration and oedema. The device was shown to be successful in managing oedema, healing complex ulcers, and in preventing ulcer reoccurrence. ${ }^{29}$ Bianchi et al. ${ }^{28}$ described the use of the JuxtaCURES device in three patients with venous ulceration, reporting improvements in quality of life, pain, well-being and cost effectiveness. Others have described improvements in pain and depression, improved skin integrity, and enhanced self-care when patients were changed to an adjustable compression wrap system from traditional compression bandaging ${ }^{29-32}$ although details of how these outcomes were measured are not clear. In a conference poster, Elson ${ }^{31}$ reported the JuxtaCURES product as useful for patients with ulceration in addressing problem with bandages, such as inconsistent pressures, bulkiness, inability to wear shoes and bandage

slippage. In another poster Elson provided a report on the JuxtaCURES system in 17 patients with chronic ulceration ${ }^{33}$ Clinicians recorded progress over six months of standard compression and six months of the use of the adjustable compression wraps; the findings indicated that all patients showed improvement in the ulcer, with favourable cost comparisons $^{33}$, although precise details of the evaluation methodology were not given. Similarly, use of an adjustable compression wrap product to replace bandaging in patients with ulceration was reported as improving patient concordance, satisfaction and independence, with staff and carers quickly able to learn the application method. ${ }^{34}$ Nugent ${ }^{35}$ also described the adjustable compression wrap devices as having a positive impact on patients' quality of life, while Oates et al. ${ }^{36}$ reported improved independence and concordance; however claims of reduced wound size and oedema are not clearly substantiated by data in the poster. ${ }^{36}$

\section{Economic implications of using adjustable compression wrap devices}

The literature search did not identify any robust economic evaluations although there is some evidence that treatment of venous leg ulcers using adjustable compression devices may provide significant cost savings $s^{35,37}$ and further research is required. An evaluation of 
the Juxta-Fit and Juxta CURES devices in 16 community-based patients with leg ulcers in the UK reported a reduction in clinical time spent on bandaging and fewer visits to patients in the community. ${ }^{38}$ In particular, a reduction in oedema and exudate meant that fewer superabsorbent dressings were required. Quality of life was also improved as patients were able to return to using normal footwear, leading to improvements in gait, stability and mobility. The author presents a calculation of possible saving of $£ 61.88$ per week as a result of using the adjustable compression wrap instead of bandaging. ${ }^{38}$

Several papers and posters suggested that self-care adjustable compression wrap devices provide cost savings associated with reduced use of bandaging materials, time saved due to quicker and easier application of the devices, application by the person or their carer, and fewer nurse visits or appointments for bandaging. ${ }^{18,28,34,35}$ Further economic advantages are also identified as the adjustable compression wrap devices can be washed, and reused over a period of 6 months, providing a cheaper option than repeated bandaging. ${ }^{22,28}$

\section{Conclusions}

In summary, the review provides some descriptive insights and anecdotal evidence for practice. However, the research evidence for the use of adjustable compression wrap devices in people with lymphoedema, chronic oedema, venous ulceration and lipoedema is very limited. Most evidence is in the form of descriptive papers, case studies, or relatively small research studies that are undertaken over a short period of time, and do not reflect the long term nature of these chronic conditions and their treatment. Despite the lack of robust economic evaluation, claims regarding cost savings are compelling, alongside the clinical evidence that an adjustable compression wrap device provides scope for improved quality of life and independence for patients with distressing long term conditions.

\section{Recommendations}

Further research is required to evaluate the effectiveness and efficacy of adjustable compression wraps in symptom management, oedema reduction and ulcer healing. Research should also focus on generating evidence to enable practitioners better understand how adjustable compression wraps influence self-management over the longer term, identifying the self-management support and education needs of individuals using the 
devices. While two studies report data on sub-garment pressures ${ }^{21,22}$, more work is required to better understand the physiological and clinical effects of the devices in patients with different conditions. For example, exploring claims that an adjustable compression wrap works in a similar way to an inelastic bandage system. ${ }^{10,11}$ Further work is required to evaluate and contrast the effect of the products with different groups: for example, individuals who undergo liposuction for lymphoedema or lipoedema ${ }^{39}$; or in people with different skin and tissue conditions such as the soft pliable tissues in someone with lipoedema, and the hard fibrotic tissues characteristic of late stage lymphoedema. ${ }^{40}$ Selfmanagement remains poorly understood and researched ${ }^{41}$, so further work is required to ensure practitioners and patients have appropriate support with new and different ways of working with patients, and are familiar with the various key aspects of self-management that complement the use of an adjustable compression wrap device.

\section{References}

1. Wounds UK Best Practice Statement. Compression hosiery. (Second edition) Wounds UK. 2015, Available from: www.wounds-uk.com.

2. Partsch, H., Moffatt, C. An overview of the science behind compression bandaging for lymphoedema and chronic oedema. In: Compression Therapy: A Position Document on Compression Bandaging. International Lymphoedema Framework in Association with the World Alliance for Wound and Lymphoedema Care, pp.12-22, 2012.

3. Kelechi, T.J., Johnson, J.J., Yates, S. Chronic venous disease and venous leg ulcers: an evidence-base update. Journal of Vascular Nursing. 2015; 33: 2, 36-46.

4. Franks, P.J., Moffatt, C.J., Doherty, D.C., et al. Assessment of health-related quality of life in patients with lymphedema of the lower limb. Wound Repair and Regeneration, 2006; 14:2, 110-118.

5. Fetzer, A., Wise, C. Living with lipoedema. Reviewing different self-management techniques. British Journal of Community Nursing, Chronic Oedema Supplement, 2015; 20:10, S14-19.

6. Deng, J., Radina, E., Fu, M.R., et al. Self-care status, symptom burden, and reported infections in individuals with lower extremity primary lymphedema. Journal of Nursing Scholarship, 2014; 47:2, 126-134.

7. Tidhar, D., Hodgson, P., Shay, C., Towers, A. A lymphedema self-management programme: Report on 30 Cases, Physiotherapy Canada. 2014; 66: 4, 404-412.

8. Miller, C., Kapp, S., Newall, N., et al. Predicting concordance with multi-layer compression bandaging. Journal of Wound Care. 2011; 20: 3, 101-112.

9. Lymphoedema Framework. Best Practice for the Management of Lymphoedema, International consensus. London: MEP Ltd, 2006.

10. Mullings, J. Juxta-Fit ${ }^{\mathrm{TM}}$ compression garments in lymphoedema management. British Journal of Community Nursing, Chronic Oedema Supplement. 2012; 17: 10, S32-7. 
11. Wigg, J. Supervised self-management of lower limb swelling using FarrowWrap. British Journal of Community Nursing, Chronic Oedema Supplement. 2012; 17: 4, S22-28.

12. Partsch, H. Compression for the management of venous leg ulcers: which material do we have? Phlebology. 2014; 29: 1S, 140-145.

13. Lund, E. Exploring the use of CircAid legging in the management of lymphoedema, International Journal of Palliative Care. 2000; 6: 8, 383-91.

14. Linnet, N., Hunt, K. Use of Juxta-Fit to reduce oedema and promote selfmanagement. Journal of Lymphoedema. 2011; 6: 2, 94-99.

15. Ehmann, S. Lymphedema compression bandages or wraps: Choosing best care for patients and for care givers. Poster presentation at SAWC, Spring Conference 2016; Atlanta, USA.

16. Lawrance, S. Use of a Velcro ${ }^{\circledR}$ Wrap system in the management of lower limb lymphoedema/chronic oedema. Journal of Lymphoedema. 2008; 3: 2, 65-70.

17. Wigg, J. A case for specialist practice. Journal of Lymphoedema. 2009; 4: 1, 72-8.

18. Wigg, J., Lee, N. Redefining essential care in lymphoedema. British Journal of Community Nursing, Chronic Oedema Supplement. 2014; 19: 2, S24-29.

19. Hobday, A., Wigg, J. Farrow Wrap: innovative and creative patient treatment for lymphoedema. British Journal of Community Nursing, Chronic Oedema Supplement, 2013; 19: 4, S24-31.

20. Langhaus-Nixon, J. An easy device for night compression in maintenance treatment of lymphoedema. Oral presentation at ESL conference, 6-8 June, Valencia, Spain, 2013.

21. Damstra, R.J., Partsch, H. Prospective, randomised controlled trial comparing the effectiveness of adjustable compression VELCRO ${ }^{\circledR}$ wraps versus inelastic multicomponent compression bandages in the initial treatment of leg lymphedema. Journal of Vascular Surgery: Venous and Lymphatic Disorders. 2013; 1: 1, 13-19.

22. Mosti, G., Cavezzi, A., Partsch, H., et al. Adjustable Velcro compression devices are more effective than inelastic bandages in reducing venous edema in the initial treatment phase: a randomised controlled trial. European Journal of Vascular and Endovascular Surgery. 2015; 50: 3, 368-374.

23. Williams, A.F., Whitaker, J. Measuring change in limb volume to evaluate lymphoedema treatment outcome. EWMA Journal. 2015; 15: 1, 27-32.

24. Lawrence, G. JuxtaCURES: An innovative method for providing compression for leg ulcer management. Wounds UK. 2014; 10: 1, 64-8.

25. DePalma, R.G., Kowallek, D., Spence, R. K. Comparison of costs and healing rates of two forms of compression in treating leg ulcers. Vascular Surgery. 1999; 33: 6, 68390.

26. Blecken, S.R., Villavicencio, J.L., Kao, T.C. Comparison of elastic versus non-elastic compression in bilateral venous ulcers: a randomized trial. Journal of Vascular Surgery. 2005; 42: 6, 1150-1155.

27. Bock, K.J. Adjustable compression wraps with overlapping straps can prevent ulcer re-occurrence in phlebolymphedema: a case series. Poster presentation, SAWC, Spring Conference 2016; Atlanta, USA.

28. Bianchi, J., Mahoney, K., Nugent, L., Kenn, D. A fresh way to treat venous leg ulcers with measured compression. British Journal of Community Nursing. 2013; 18: 4, S3440. 
29. Davies, L. A novel compression device improving patient quality of life (JuxtaCURES ${ }^{\mathrm{TM}}$ ). Poster Presentation, Wounds UK Conference. 2013.

30. Dowsett, C., Elson, D. Meeting the challenges of delivering leg ulcer services. Wounds UK, 2013; 9: 1, 90-5.

31. Elson, D. The evolution of compression devices for venous leg ulcers. Poster Presentation EWMA May 2013.

32. Dowsett, C. Treatment and prevention of recurrence of venous leg ulcers using RAL hosiery. Wounds UK. 2011; 7: 1, 115-9

33. Elson, D. The $21^{\text {st }}$ Century approach for cost effective healing of venous leg ulcers. Poster Presentation, Wounds UK Conference 2012.

34. Elvin, S. Cost efficacy of using Juxta-CURES ${ }^{\mathrm{TM}}$ and UCS ${ }^{\mathrm{TM}}$ Debridement cloths. Journal of Community Nursing. 2015; 29: 2, 25-28.

35. Nugent, L. Juxta-CURES: compression for healing venous ulcers. British Journal of Community Nursing. 2013; 18: 5, S40-45.

36. Oates, L., Ranton, A., Roberts, D. A simple measureable graduated compression device (Juxta CURES ${ }^{\text {TM}}$ ), Poster Presentation. Wounds UK Conference 2013.

37. NICE. The Juxta-CURES adjustable compression system for treating venous leg ulcers. Available at: https://www.nice.org.uk/advice/mib25/chapter/Evidence-review, NICE Advice MIB25, March 2015. [accessed $22^{\text {nd }}$ April 2016]

38. Wick, G. An alternative to compression bandaging in venous leg ulcers. Journal of Community Nursing 2015; 29: 4, 40-46.

39. Forner-Cordero, I., Szolnoky, G., Forner-Cordero, A., et al. Lipedema: an overview of its clinical manifestations, diagnosis and treatment of the disproportional fatty deposition syndrome - systematic review. Clinical Obesity. 2012; 2: 3-4, 86-95.

40. International Society of Lymphology (ISL). The diagnosis and treatment of peripheral lymphedema: 2013 consensus document of the International Society of Lymphology, Lymphology. 2013; 46: 5, 1-11.

41. Long Term Conditions Alliance. Gaun Yersel. Being human: the self-management strategy for long term conditions in Scotland. October 20-8, Edinburgh, Scottish Government. 